\title{
Los inicios del seguro privado en Guipúzcoa: de las sociedades de socorros mutuos a las primeras compañías (1842-1914)
}

\author{
Carlos larrinaga - Carmez lázaro \\ Universidad de Deusto
}

\section{INTRODUCCIÓN}

La evolución del sector asegurador en España presenta dos períodos claramente diferenciados, cuya frontera se sitúa en el inicio de la industrialización que en España se caracterizó por su tardio inicio respecto de otros paises europeos.

El primer período es anterior al proceso industrializador y se distinguió por el protagonismo del llamado seguro marítimo ', con un marco legislativo regido por el Derecho canónico que prohibía tanto la usura como la productividad del capital pero que admitía la cláusula de seguro, porque el pago por el riesgo sufrido y el interés económico se consideraban legítimos. Como consecuencia de ella el comercio, en auge desde finales de la Edad Media, encontró en el seguro una fórmula especulativa de uso de capitales, a la vez que podia seguir eludiendo la legislación canónica ${ }^{2}$.

El empleo de fórmulas primitivas para la asociación de capitales, como la commenda y la compañía comanditaria por acciones ${ }^{3}$, incapacitaban el desarrollo de un negocio estable de seguros.

Matilla y Frax Rosales (1993), pág. 85, mencionan la importancia que tuvieron en esta etapa las Ordenanzas del Consulado de Bibao de 1737, pero teniendo en cuenta que durante toda la Edad Moderna la base de organización de los seguros fue personalista y casi exclusivamente para el seguro de transporte, especialmente maritimo.

2 Todo ello no hizo sino dificultar la asociación de capitales, elemento indispensable para el desarrollo de una economia moderna. Con la llegada de la Contrarreforma y el Concilio de Trento, en el siglo xvi, desapareció la necesidad de eludir la legislación canónica, eliminándose los caracteres del préstamo marítimo, si bien el seguro se mantuvo, porque podia seguir respondiendo a las necesidades del comercio.

La commenda permitia que un comerciante viajero contara con los recursos suficientes para comprar o vender mercancias en sus viajes. Pero con la sedentarización del comerciante y su progresiva conversión en negociante, esta fórmula perdió interés y fue sustituida por otras más atractivas para la nueva situación. 
Ahora bien, tampoco se puede olvidar la fuerza que la organización gremial tuvo, habilitando ciertas posibilidades de previsión entre sus asociados. No obstante, la coyuntura adversa del siglo XVII y las perpetuas quiebras del Estado y los municipios esquilmaron continuamente las arcas gremiales, debilitando su poder $y$, por supuesto, sus posibles prestaciones.

En cuanto al segundo período del desarrollo asegurador, podemos decir que evolucionó paralelo al proceso de industrialización. Con el progreso del capitalismo aumentó la inseguridad de la clase trabajadora, causada por unas ciudades sin infraestructuras - debian absorver una mano de obra emigrante masiva en espacios de tiempo muy reducidos- y por unas jornadas de muchas horas laborales a cambio de salarios bajos que no permitian ahorrar y prevenir un posible futuro de incapacidad ocupacional. La exposición a peligros era continua, con el consiguiente riesgo de accidente o enfermedad. Además, con la llegada de la vejez, el trabajador era calificado de «inservible". Igualmente, a partir de este momento la familia "extensa" pierde su fuerza protectora ante los riesgos ajenos a ella ${ }^{4}$. El antiguo asalariado, separado de los medios de producción, estaba bajo la tutela del patrono burgués que se ocuparía de él mientras fuera productivo y bajo la tutela de la beneficiencia liberal cuando perteneciese a la masa de indigentes incapacitados.

Mientras, la burguesía buscaba su concordia social con el poder establecido y con la nobleza ${ }^{5}$, atendiendo en un segundo plano e incluso ig-

La Compañía comanditaria por acciones apareció a finales del siglo xvIII, estableciendo la existencia de unos socios que aportaban capital a la empresa, sin contraer por ello obligaciones mercantiles. Puede considerarse como un paso intermedio entre la compañía personalista y la anónima, que prevaleció en el sector asegurador hasta 1929, año en el que por R.D. el Ministerio de Hacienda les prohibió el ejercicio del seguro.

4 Cipolla (1992), pág. 340: "La unidad familiar preindustrial es tradicionalmente una instituciónı numerosa, de carácter patriarcal, que amén de la función básica de procrear, criar y educar nuevas generaciones, satisface dentro de su propio ámbito funciones económico-productivas y numerosas tunciones que hoy llamariamos de seguridad social (el cuidado de los miembros enfermos y de los viejos). La familia de la sociedad industrial es una unidad numéricamente restringida, relativamente menos estable y más limitada en el tiempo y con funciones en el mundo agricola que estaban atribuidas a la familia".

s PESET (1978), págs. 187 y ss.: «... en este momento, en toda Europa, la burguesia procura pactar con las fuerzas que aspira a derrotar, nobleza y burguesia nadan de la mano, en busca de dinero y honores, hacia mediados del siglo $x \mid x, y$, por tanto, en busca de una primera concordia entre las dos clases sociales, beneficiencia eclesiástica y estatal. En Francia es la figura de THIERS quien plasma mejor esta actitud y no es raro que reciba los elogios más encomiásticos su tempo. ral contemporización. THIERS admitía una caridad privada, la pública, más prudente y limitada, que debe completar aquella e ir con cuidado para no provocar derroche y ociosidad. El Estado tiene muchos gastos, no puede evitarse excesos que tal vez, en lugar de evitar, aumentarian la mendicidad, un pacto con la Iglesia es conveniente". 
norando las necesidades de previsión de la clase obrera. El obrero se vio obligado a no abandonar la herencia mutualista, empleándose como una contestación funcional frente a la proletarización de las clases trabajadoras. Desde este punto de vista que lo considera ideológicamente neutro, su único interés era que revelaba un mayor afán por organizarse.

Este era el marco general de principios de siglo cuando desde el poder se inició el camino hacia la previsión social, dando a entender que no se debia a presiones del obrerismo. Asi, frente a esta iniciativa, el sector del seguro privado trató de defender sus intereses, principiando el camino hacia la modernización legislativa y empresarial, sin perder parte en el jugoso negocio de la previsión.

1908 fue el año clave para dilucidar lo que constituyó el posterior desarrollo de la previsión en España, ya que en ese año se tramitaron en el Parlamento dos leyes fundamentales casi al mismo tiempo. La primera fue la Ley Orgánica de 27 de febrero de 1908, fruto de la cual fue el Instituto Nacional de Previsión. Las protestas obreras, la crisis política de $1898{ }^{6}$ y el aumento general de la conflictividad social fueron suficientes motivos para que las clases dominantes iniciaran el camino de la institucionalización de la previsión. En efecto, la monarquía de Alfonso XIII aceptó sin titubeos un modelo intervencionista de Estado tutelar que anulaba las tensiones entre la burguesía y el proletariado para garantizar la supervivencia del sistema y, sobre todo, la suya propia.

El segundo hito legislativo importante de ese año fue la Ley General de Seguros de 4 de mayo. Hasta la creación de ésta, el Código de Comercio vigente era el principal marco legistativo al que se ajustaba el sector? Como consecuencia de la debilidad estructural de las compañias y la consiguiente falta de respuesta ante los clientes, los legisladores sobre seguros de comienzos del siglo $x x$ atendieron preferentemente los aspectos relacionados con la formación de las compañías para preservar un aso-

\footnotetext{
6 MARtinez (1990), págs. 262 y ss.: “Gracias a la coyuntura crítica de 1898, lograron audiencia y apoyo en algunos politicos de la Restauración los que insistian en la necesidad de adelantarse a la revolución con la reforma, o de suavizar, mediante ella, la lucha de clases. Recordemos que la iniciativa se habia generado entre los intelectuales de clases medias y algunos profesionales ganados por el krausismo, el positivismo y el utilitarismo, a los que habia que sumar los católicos sociales, alentados por la Rerum Novarum".

7 Matulla (1986), pág. 9, afirma que el primer desarrollo del seguro está netamente condicioinado a la aparición del Código de Comercio de 1885, el cual permitió que las sociedades de seguros contribuyeran por simple inscripción tanto si adoptaban viejas fórmulas - colectivas o comanditarias-, como si se constituian como anónimas. Sin embargo, faltaba crear un clima socio-politico estable que permitiera la supervivencia del desarrollo económico.
} 
ciacionismo de capitales sólidos. La correcta regulación del sector permitió que el asegurado no corriera riesgos verosimiles de quedarse sin defensa ante la falta de coberturas en épocas de crisis ${ }^{8}$.

\section{PROPIEDAD Y SEGUROS MUTUOS}

El establecimiento de sucursales que representaban y gestionaban pólizas de compañías aseguradoras nacionales y extranjeras en Guipúzcoa se produjo paralelo al resto del pais. Sin embargo, existieron asociaciones de carácter mutuo creadas en el periodo de entreguerras carlistas ${ }^{9}$.

En el caso guipuzcoano tenemos noticias de dos mutuas propiamente provinciales que aseguraban contra incendios y que en buena medida, pueden ser consideradas como antecedente de las primeras compañías de seguro privado de la provincia surgidas a finales del siglo XIX y que van a ser objeto de atención en este apartado.

A diferencia de las compañías de seguro privado, las asociaciones creadas a mediados del siglo XIX sólo pretendían cubrir las calamidades y pérdidas ocasionadas por los incendios mediante una compensación económica. El horror que despertaba el fuego y sus efectos devastadores lievaron a la aparición de asociaciones de carácter mutuo con fines previsores, en las que cada socio propietario asegurado era a su vez asegurador ${ }^{10}$. Sus objetivos distaban mucho de atender riesgos personales de los ase-

\& La nueva reglamentación ejerció un control tal que, entre otras normas, disponia la obligación de un desembolso previo minimo del $25 \%$ del capital suscrito, asi como el envio a la Inspección de resguardos que certificasen inversiones en valores públicos, industriales o materiales, previaniente aceptadas por el propio Ministerio. Sin embargo, las inestables compañias comanditarias por acciones mantuvieron su parcela en el negocio hasta el mencionado R.D. de 1929.

$9 \quad$ La Real Orden de 1839, que reconocia el derecho a constituir libremente sociedades cuyo fin fuese "auxiliarse mutuamente en sus desgracias, enfermedades, etc. o reunir el producto de sus economias con el fin de atender a sus necesidades futuras", pudo ser el marco legal en el que se insertaba, la creación de estas mutuas. Se trataba de tiempos de turbulencias políticas y desde el poder se contemplaba con recelo el asociacionismo porque podía enmascarar propósitos poli. ticamente contrarios.

El reconocimiento legal pleno del derecho de asociación, sin otro limite que el respeto a la moral publica, no se produjo hasta la aprobación de la Constitución de 1868.

10 El Reglamento para la Sociedad de Seguros Mutuos de Casas y Caserios de Guipuzcoa (1848) define en su articulo 2. como objeto de la Sociedad la garantia mutua infalible proporcionada por la fórmula de que los socios son a su vez asegurados, obligándose, con hipoteca de las fincas aseguradas, a los daños causados por los incendios a fin de indemnizarse reciprocamente su importe a prorrata del capital asegurado exceptuando los estragos generados por incendios procedentes de fuerza armada. 
gurados, ya que era la propiedad mobiliaria lo único que en todo momento se atendia como objeto de seguro de las asociaciones.

Las mutuas no aseguraban fábricas ni edificios públicos o de uso municipal, lo que se tradujo en una contratación encaminada hacia pólizas del seguro privado (nacional o extranjero) ${ }^{11}$, por no encontrar en las mutuas suficientes garantías para la envergadura del valor de un edificio público. Tampoco se pensó en la vía mutualista como una posibilidad para asegurar bienes públicos que podian resultar excesivamente costosos y pudieran hacer peligrar el difícil equilibrio que debia mantener la mutua para un correcto funcionamiento que garantizase reposición patrimonial a asegurados más modestos. En concreto, el reglamento de la Sociedad de Seguros Mutuos de Guipúzcoa excluía edificios públicos y fábricas ${ }^{12}$.

De las dos mutuas mencionadas, la primera en crearse fue la "Asociación de Seguros contra incedios de San Sebastián". El 21 de marzo de 1842 un grupo de seis propietarios ${ }^{13}$ de San Sebastián firmaban una carta dirigida al Ayuntamiento constitucional de la ciudad solicitando su apoyo para la creación de una asociación de seguros contra incendios. La aprobación del Consistorio suponia un importante aval con vistas a la captación de asociados ${ }^{14}$.

Eran los años posteriores a la Primera Guerra Carlista y, por lo tanto, los años en que se produjo el definitivo traslado de las aduanas a la frontera. La ciudad vivía un antagonismo permanente con el resto de la provincia que asistía al deterioro de las estructuras propias del Antiguo Régimen.

Los objetivos económicos de la burguesía de San Sebastián de carácter eminentemente comercial sólo buscaban la protección de sus intereses desde su presencia al frente del Regimiento, superando a cualquier precio el grave deterioro en que se hallaba la ciudad. Los firmantes de la mayoria de los documentos de la Sociedad ocuparon cargos de responsabilidad a lo largo del siglo xIx, a pesar de que en el plano profesional no alcanza-

"Archivo Municipal de San Sebastián (AMSS), Sec. A., Neg. 16, Ser. I, Lib. 142, Exp. 2: La "Póliza de la compañia de seguros La Urbana" aseguraba en 1867 contra el incendio, el rayo, la explosión de gas y de los aparatos de vapor el edificio del Ayuntamiento de San Sebastian de un valor de 240.000 reales de vellón por una prima anual de 1447 reales.

12 Reglamento... (1848), art. 58.

13 Bartolome francisco oe lopetedi, jose Maria de ahumada, ignacio alzola, francisco Javier Gasende, Joaquin A. de Elosegui y José francisco de Arzac.

14 AMSS, Sec. A, Neg. 16, Ser. I, Lib. 142, Exp. 2. Tambièn contó con la aprobación del Jefe Político de la Provincia. 
sen la pujanza de nombres como Brunet, Berminghan, Collado o Echagüe en instituciones de la ciudad como el Consulado. Por ello pueden ser catalogados como pequeños comerciantes que buscaban en la posesión de bienes raíces una seguridad futura para sus capitales, escapándose del alto riesgo de las operaciones mercantiles ${ }^{15}$.

La Asociación se presentaba como una iniciativa local en el seguro contra incendios para las casas de la ciudad, tanto intramuros como extramuros, si bien en muchos casos éstas no carecían de seguro, ya que éste recaía en manos de extranjeros con el consiguiente beneficio que ello suponía. Sin duda, los propietarios de la ciudad que apoyaron la iniciativa aseguradora local consideraban esto como algo inadmisible ${ }^{16}$.

De esta forma, con la aprobación del Consistorio se puso en marcha la creación de una reglamentación ${ }^{17}$ que regía las medidas a tomar para la prevención de incendios, así como la actuación a seguir por el colectivo de ciudadanos en caso de ocurrir un siniestro. El apartado de la prevención regulaba el almacenamiento de todo tipo de materias inflamables y el mantenimiento y disposición de chimeneas, hornos, estufas, etc., cuyo incumplimiento se tendría en cuenta al calificar las causas del incendio. Las bombas empleadas para apagar los fuegos exigian un grupo heterogéneo de personas denominadas arquitectos, maestros de obras y maniobreros, a quienes la Sociedad pagaba por sus servicios si la casa siniestrada estaba asegurada. Después el socio debía abonar a la Sociedad la mitad del coste del servicio de incendios. Ahora bien, cuando la casa no estaba asegurada era el propio Ayuntamiento el encargado de pagar dichos servicios. La creación de la mutua atendía en primer lugar ese pago por los servicios prestados a quienes extinguían el incendio y después pasaba a ocuparse de la reposición de los bienes materiales perdidos, así como de la custodia de los salvados.

En 1857 la Asociación contaba con un capital responsable de veinte millones de reales de vellón en edificios de la propiedad de socios que, según palabras de los directores, proporcionarian una garantía mutua infalible. Sin embargo, entre 1860 y 1863 la Junta General de la Sociedad decidió que la

is ApARICIO (1991), pág. 266.

16 AMSS, Sec. A., Neg. 16, Ser. I, Lib. 142, Exp. 2: "Muchas son las casas de esta ciudad que están aseguradas, y lástima causa de considerar, que pudiendo llenar en el mismo pueblo ese objeto, vayan los aprovechamientos al extranjero".

17 AMSS, Sec. A., Neg. 16, Ser. I, Lib. 142, Exp. 2: "Reglamento para casos de incendio de esta ciudad de San Sebastian acordado y redactado por disposición del llustre Ayuntamiento Constitucional, y la Dirección de la Sociedad de Seguros mutuos en cumplimiento del articulo 17 del reglamento orgánico de la misma Sociedad". 
contribución de las fincas aseguradas debía ser proporcional al riesgo que presentaban. Hay que tener en cuenta que la finalidad de un contrato de seguro es prevenir o reparar las consecuencias patrimoniales desfavorables o necesidades que un riesgo pueda desencadenar, por lo que el funcionamiento mutualista establecia unas obligaciones que cabría calificar de muy simples e insuficientes ante una sucesión continuada de riesgos, de suerte que en un corto espacio de tiempo agotaría las reservas acumuladas a través de las contribuciones de los asociados. Un periodo de sucesivas catástrofes podía ser suficiente causa para restringir las coberturas del seguro si no se tomaban con antelación medidas que lo impidiesen. De esta manera, una contribución proporcional al riesgo que se deseaba asegurar era una medida imprescindible para que un futuro adverso no dejase a la Sociedad de Seguros Mutuos sin capacidad de respuesta ante sus asegurados.

No resulta extraño que en los informes que la Sociedad obligaba a realizar sobre el siniestro que ocasionaba el incendio se detallaran las posibles causas y la actuación de quienes habitaban en la propiedad, ya que la actuación con dolo o posible intencionalidad podía estar presente, por lo que sólo quedaban fuera de toda sospechas quienes estaban ausentes del edificio en el momento en que se producía el primer fuego. La insistencia del Ayuntamiento para controlar la continua limpieza de las chimeneas y tiros es manifestada una y otra vez a través de bandos, pudiendo ser multados aquellos que no cumplieran las pautas establecidas a este respecto. La Asociación era una previsión para reparar los males posteriores al incendio. La atención previsora por parte de los asociados que evitara riesgos, exigida desde el Ayuntamiento, era la lógica consecuencia ante el temor a un aumento desmesurado de este tipo de siniestros.

A finales del siglo XIX la Sociedad tuvo constancia de algunas bajas entre sus asociados por causas ajenas a éstos, pasando a ser bienes asegurados en compañías de seguros ${ }^{18}$. Posiblemente nos encontramos ante un mercado claramente diferenciado en el que las compañias abarcaban las coberturas dirigidas a edificios públicos, fábricas y viviendas de lujo y la mutua se centraba en la cobertura para reponer lo imprescindible en la mayoría de las viviendas de la ciudad.

\footnotetext{
is AMSS, Sec. A, Neg. 16, Ser. I, Lib. 142, Exp. 2: Una circular emitida por la Sociedad a sus socios en 1889 apuntaba lo siguiehte: "Tampoco puede decirse que se ha sacado casas de la Sociedad, pues una y otra parte de otra, unicas salidas, no to fueron por voluntad de los Sres. Socios sus dueños, sino porque vendidas a quienes tenian ya de antes asegurados los géneros de la una y la parte de la otra en Sociedades a prima fija, juzgaron lo más natural tener reunidas en las pólizas de estas las propiedades que adquirieron".
} 
La segunda mutua que nos ocupa es la «Sociedad de Seguros Mutuos contra incendios de Casas y Caseríos de Guipúzcoa». Nació en el verano de 1847, después de que la Junta séptima de la Diputación de Guipúzcoa de ese mismo año, celebrada en Oñate, contara con la propuesta hecha por el Procurador a Juntas Generales y representante de Rentería, Luis de Sorondo ${ }^{19}$, quien llamó la atención sobre "la necesidad de plantear en el pais asociaciones mutuas sobre incendios" 20 y se reveló como un claro defensor de las mismas por sus fines filantrópicos. No hay que olvidar que la implantación de una sociedad capitalista estaba estrechamente ligada a la potenciación de la liberalización de la tierra y de la propiedad privada y el perfeccionamiento de las relaciones juridicas de la misma. El interés hacia el seguro de incendios surgió por la adquisición de una propiedad y su deseo de salvaguarda ante contingencias imprevisibles ${ }^{21}$.

No tenemos noticia de fecha más temprana sobre asociacionismo con objetivos dirigidos a la previsión de riesgos que afectasen a bienes muebles o inmuebles de propiedad familiar y en muchos casos de carácter rural. Al respecto, es importante recordar que hasta el 28 de febrero de 1839 no se reconoció legalmente el derecho de asociación con finalidades mutualistas $^{22}$.

En la propuesta del miembro de las Juntas Generales se observa una iniciativa que no desea desmarcarse de la legalidad vigente, pues sólo pretendia crear una asociación inscrita en los parámetros legales de un asociacionismo con fines de auxilio mutuo en las desgracias, enfermedades o necesidad futura, reuniendo para ello el producto de sus economías. En este caso, la Diputación apoyó con una ayuda económica en concepto de préstamo el primer paso para la puesta en marcha de la creación de una asociación que proporcionara la cobertura necesaria ante el riesgo de incendio, el cual podia suponer a menudo la pérdida total o de la mayor

\footnotetext{
19 Véase Cruz Mundet (1991) y Barcenilla (1992). Luis de Sorondo pertenecía a una familia de propietarios medios de tierras que durante la segunda década del siglo XIX pudo acceder a la compra ventajosa de tierras que pertenecian a pequeños propietarios, adquiriéndolas por la milad o por la tercera parte de su valor. La acumulación de rentas de este tipo permitió a ésta y a otras familias de Renteria que siguieron procesos similares un acopio de capital tal que les sirvió de base para la instalación de las primeras tábricas modernas a lo largo de la segunda mitad de dicho siglo.

4. Las Juntas Generales de 1847 encargaron a la Diputación que procurase fomentar en la provincia el espiritu de asociaciones mutuas sobre incendios.

2) La sociedad liberal y capitalista en su versión moderna que se fue implantando en España a mediados del siglo xIx tenía como uno de sus pilares básicos el concepto de propiedad, argumentado tilosóficamente primero por LoCKE y más tarde por HEGEL, entre otros.

22 GaRRIDO y COMAS (1994). pág. 23.
} 
parte de las posesiones de una familia o el fruto del trabajo de muchos años. El incendio era un riesgo que carecía en esta época de servicios de extinción de incendios profesionales, aunque sí existía una colaboración vecinal que a todas luces era insuficiente, por lo que la probabilidad de un seguro que implicaba una remuneración económica contra el que era el riesgo más frecuente de las propiedades de la época no era una idea desdeñable, siempre que contara con una gestión administrativa aceptable.

Por ello, el apresurado primer paso, después de la propuesta realizada en las Juntas Generales, lo dieron en julio del mismo año «algunos propietarios" de la provincia. Suscribieron el reglamento de la asociación treinta personas, por lo que los promotores de la misma consideraron que el proyecto tenía la entidad suficiente como para que la Diputación amparara el mismo ${ }^{23}$, pudiendo de esta manera dar cabida a la propuesta hecha por el representante de Rentería en las Juntas de Oñate.

La cobertura del riesgo se concebia todavía bajo un punto de vista solidario. No se esperaba más beneficio que el poder abonar indiscriminadamente una cantidad que mitigase las calamidades del siniestro entre los asociados de la mutua que se vieran afectados por un incendio devastador. La cantidad asegurada eran las tres cuartas partes del valor de la propiedad establecida por acuerdo mutuo entre la Sociedad y el asegurado ${ }^{24}$. Ahora bien, si un socio deseaba abandonar voluntariamente la mutua perdia todos sus derechos sobre lo que había contribuido y en caso de dolo por parte del asegurado la Sociedad no cancelaba sus obligaciones con el mismo ${ }^{25}$.

La Sociedad de Seguros Mutuos no tuvo desde sus comienzos una constitución propia de sociedad ni una organización administrativa sólida, porque los inicios no estuvieron acompañados de un incremento masivo en el número de los asociados. Sus representantes reflejaban su preocupación ante cualquier desgracia, ya que con el pago de las cuotas por parte

2:3 Archivo General de Guipuzcoa (AGG), JD-IT-989a: «Hubiera sido por lo tanto reprensible que la comisión directiva de la sociedad dejara de noticiar a V.S. su instalacion, y al ponerla en su conocimiento toma la libertad de indicar a V.S. que seria quizas muy conveniente el que nos acercásemos a V.S. de pensarnos para que nuestros trabajos correspondan á las esperanzas que concibieron los socios al honrarnos con su elección".

24 Reglamento... (1848), art. 3...

25 La mutua se creaba con la finalidad de atender la necesidad de una falta de prevención contra los inevitables incendios que a menudo sufrian quienes poseian propiedades, por lo que debia fomentar la correcta actuación de quien era asegurado, asi como una constante advertencia a quienes pudiesen ver en el seguro de sus propiedades posibles fuentes de ingresos. 
de las personas asociadas durante los primeros meses de vida de la sociedad era imposible crear un fondo de grandes cantidades que permitiera cubrir los riesgos de un incendio en una propiedad. Es evidente que la asociación no popularizó sus servicios de forma masiva desde el principio.

La preocupación de las primeras cartas del presidente de la Sociedad se refería a una minoría de asociados cuyas cuotas depositadas en el fondo común de la misma eran insuficientes para proporcionar una cobertura aceptable ante un incendio de una propiedad de "mucho valor». Esta especificación revela el carácter de la mayoria de las propiedades hasta entonces aseguradas en la asociación. Si el carácter de los bienes asegurados hubiese sido más heterogéneo, siendo minoria las propiedades valiosas, esta inquietud hubiera sido injustificada.

En marzo de 1848 la gestión de la asociación podía abandonar sus temores gracias a que el número de socios se habia elevado a 602 . Dicha cantidad, sumada a los socios que deseaban ingresar en la Sociedad a partir de su constitución estable, permitía que en caso de siniestros se pudiera ofrecer contraprestaciones aceptables a los asociados. El incremento de éstos pudo ser una consecuencia lógica de la divulgación que desde finales de 1847 había llevado a cabo la incipiente asociación a través de la propia Diputación, mediante el envio gratuito de ejemplares del reglamento de la entidad a distintos alcaldes, párrocos y diputados para que procediesen a su reparto. No obstante, después de la primera junta general ordinaria, celebrada en Villafranca el 28 de mayo de 1848, se hicieron modificaciones en su reglamento.

La Sociedad reunía en junta anual a todos los asociados de la misma, pero no se permitia emitir voto alguno sobre las decisiones a adoptar a quienes no tuvieran fincas aseguradas por valor de diez mil reales de vellón ${ }^{26}$. El carácter censitario de la medida es propio de la época en la que aquélla se creó, permitiendo acudir sin voto alguno a quienes tuvieran una propiedad que no alcanzase dicha cuantía. Con todo, desconocemos el porcentaje aproximado de socios que contarian con derecho a voto, si bien es de suponer que no sería muy elevado el numero de posibles asociados con propiedades que superasen la cantidad mencionada.

Por otro lado, mientras la cabeza visible de la gestión de la misma pertenecia al presidente, la gestión económica era labor del tesorero, el cual contaba con un cuerpo de inspectores repartidos por toda la provincia para

26. Reglamento... (1848), art. 28. 
ejecutar el cobro de las cuotas y el reparto de las tarjetas que certificaban que la propiedad estaba asegurada ${ }^{27}$.

Cada inspector se hacia cargo de un distrito, existiendo más de uno en cada comarca, y la dirección se ocupaba de divulgar los nombres de los inspectores, asi como sus sustituciones por cese o defunción. Concretamente, la provincia fue dividida en veintitrés distritos en el año 1848.

Por otra parte, la estructura básica de la gestión económica estaba estipulada en el reglamento, pero cada junta general decidía sobre la cuantía de los pagos, exigiendo a los asociados de reciente aparición una cantidad superior. Además, el fondo de la Sociedad sufría todos los años el desgaste por los pagos a los asociados cuyas propiedades eran victimas del fuego. Con la ampliación del número de asociados se produjo el aumento de los riesgos ante posibles incendios, por lo que no se pudo renunciar a exigir una cuota ligeramente superior a quienes estrenaban su asociacionismo cada año para cubrir ese incremento de riesgos que su propia afiliación implicaba.

\section{EL SEGURO, UN NEGOCIO INCIPIENTE (1886-1900)}

Como es sabido, el comienzo del proceso de modernización de Guipúzcoa se suele situar generalmente en 1842, precisamente a raíz del establecimiento de las aduanas en la costa y en la frontera. Desde entonces se inició una tendencia creciente de desarrollo económico únicamente interrumpida por el estallido de la Segunda Guerra Carlista. No debemos olvidar que, junto al decreto del traslado de aduanas, la política económica de los progresistas durante el Bienio 1854-56 y la llegada a San Sebastián del Ferrocarril del Norte de España en 1864 contribuyeron decididamente a semejante expansión. Prueba de ello fueron las primeras papeleras modernas de la comarca de Tolosa, ya desde los años cuarenta; el notable desarrollo de la industria textil, sobresaliendo las instalaciones de Oria, Rentería y Vergara; las empresas siderúrgicas de Lasarte y Mondragón o la producción armera de Eibar. Paralelamente, en estos años tiene lugar el nacimiento de la banca moderna guipuzcoana, fundándose en 1864 el Banco de San Sebastián. Es más, incluso diversas entidades foráneas llegaron a instalar sus sucursales en esta ciudad. Además, en fachada.

27 Estas eran repartidas previo pago y se aconsejaba su colocación en un lugar visible de la 
1870 se empezó a trabajar en las obras de mejora del puerto de Pasajes, por entonces en muy malas condiciones ${ }^{28}$.

Con todo, este despertar del crecimiento económico guipuzcoano se vió bruscamente suspendido por la Segunda Guerra Carlista. Efectivamente, se debió esperar a 1876 para empezar a recuperar el ritmo perdido. Fue a partir de entonces cuando realmente comenzó el proceso industrializador de la provincia. Tal es así que éste se convirtió, en opinión del profesor Castells, en el auténtico motor de la modernización de Guipúzcoa ${ }^{29}$.

Es precisamente en este contexto de modernización general de la sociedad guipuzcoana donde debemos situar la aparición de las primeras compañías de seguros. A tenor de los datos recogidos en el Registro Mercantil de la provincia, para la década de los años ochenta del pasado siglo contamos ya con algunas sociedades dedicadas a esta actividad. En concreto, hablamos de compañías que no sólo trabajaban el seguro contra incendios, sino también el de vida, enfermedad, accidente laboral, etc. Así, mientras la "Asociación de Seguros contra incendios de San Sebastián» y la "Sociedad de Seguros Mutuos contra incendios de Casas y Caserios de Guipúzcoa" únicamente aseguraban propiedades, y bajo la forma de mutuas, en este momento la actividad aseguradora empezaba a diversificarse, además de canalizarse a través de otro tipo de fórmulas. Con todo, realmente apenas encontramos compañías consagradas al seguro privado propiamente guipuzcoanas, y más concretamente donostiarras, hasta comienzos de este siglo. En este sentido, podemos establecer la siguiente división entre las compañías que operaron en Guipúzcoa en el campo de los seguros entre finales del siglo pasado y comienzos de éste. Por un lado estarían aquellas sociedades en las cuales los seguros constituian un renglón más de su actividad, siéndonos en la mayor parte de los casos muy dificil establecer la importancia del mismo dentro del volumen total de sus operaciones. Por otro, debemos señalar aquéllas que eran compañias de seguros propiamente dichas. Ahora bien, dentro de éstas es menester distinguir entre las donostiarras en sí ${ }^{30}$, las sucursales de otras sociedades y aquéllas

${ }^{24}$ Para esta primera etapa del desarrollo económico guipuzcoano, véase GáRATE (1976).

23 CASTELLS, (1987).

(40) Con esta denominación nos estamos refiriendo a aquellas compañías domiciliadas en San Sebastian (porque no hay ninguna que tenga su sede social fuera de la capital donostiarra) y que actuaban por su cuenta, sin depender de ninguna otra sociedad (en calidad de sucursal o de re. presentante), independientemente del origen del capital. 
que actuaban en representación de otras, generalmente grandes compañías nacionales. Así lo podemos observar en el cuadro n. $\stackrel{1}{1}$.

Cuadro n.. 1. Sociedades dedicadas al seguro privado registradas en Guipúzcoa entre 1886 y 1914

\begin{tabular}{lll}
\hline AÑO & SOCIEDAD & TIPO DE ACTIVIDAD \\
\hline 1886 & Pagola y Sagasti & representación \\
1887 & El Fénix, de París & sucursal \\
1888 & R. Instander y cía. & representación \\
1892 & Nau y Palmarini & actividad secundaria \\
1894 & F. Echevarría e Hijo & actividad secundaria \\
1895 & Hijos de Lapazarán & representación \\
1901 & El Norte & cía. donostiarra \\
1904 & Franco-Inglesa & cía. donostiarra \\
1910 & La Previsión Popular & cía. donostiarra \\
1912 & Banco Español del Rio de la Plata & actividad secundaria \\
1912 & Sagasti e Hijo & cía. donostiarra \\
1914 & A. Vildósola y cía. & representación \\
\hline
\end{tabular}

FUENTE: Registro Mercantil de Guipúzcoa, libros 1-25.

Podemos detectar que 1900 supuso un claro punto de inflexión en lo que a la actividad aseguradora se refiere. Fue entonces cuando surgieron compañias propiamente guipuzcoanas dedicadas enteramente a los seguros. Desde este punto de vista, pues, podemos hablar de dos subperíodos bien diferenciados.

En el primero de ellos encontramos sociedades dedicadas al seguro en exclusiva, pero no hay compañias propiamente independientes. Efectivamente, hay un predominio de aquéllas que actuaban en representación de otras. Fue el caso de "Pagola y Sagasti», "R. Instarder y cía.» y de "Hijos de Lapazarán".

"Pagola Sagasti" fue fundada en 1886 por los comerciantes José Pagola y José Antonio Sagasti con el objeto de llevar a cabo "la esplotación de la comisión o representación de la Compañía aseguradora Unión y el Fenix Español" ${ }^{31}$. Con un capital social de 15.000 pesetas, éste fue

3) Registro Mercantil de Guipuzcoa (RMG), Lib. 4, Hoja 27. 
aportado integramente por José Antonio Sagasti, comprometiéndose ambos socios a mantener la sociedad hasta que durase la representación. Ignoramos su fecha de disolución, pues no consta en el Registro, pero sabemos que en 1895 se fundó "Hijos de Lapazarán" para representar a las compañias de seguros que así lo desearan, en especial a "La Unión y ef Fénix Español». María y Antonio Lapazarán Ibarra eran hijos de Antonio Lapazarán, quien también se habia dedicado al negocio asegurador ${ }^{32}$. Detectamos ya una cierta tradición de traspasar dicho negocio, al parecer porque empezaba a resultar rentable. Al respecto debemos recordar que el mismo José Antonio Sagasti fundó en 1912 junto a su hijo Ignacio Juan Antonio Sagasti la sociedad "Sagasti e Hijo».

Por su parte, “R. Instander y cía". fue creada en 1888 por Rosa Instander y el comerciante Eugenio Gabilondo con el fin de gestionar seguros contra incendios en representación de la sociedad "La Urbana". Con una duración indefinida y con un capital de 1.000 pesetas puesto a medias, Rosa Instander prestó la fianza de 20.000 ptas. exigida por «La Urbana".

Un caso bien distinto lo constituye la instalación de «El Fénix» de París en la capital guipuzcoana. En efecto, el 11 de julio de 1887 M. Montesquion y Du Presnay, presidente y director respectivamente del consejo de administración de dicha compañía, otorgaron un poder a Santiago Echave en calidad de agente general en el distrito de San Sebastián para representar los intereses de la sociedad en la zona, recibir las proposiciones de seguros, determinar las primas y fijar las condiciones de las pólizas ${ }^{33}$. Este constituye el primer caso de lo que podemos considerar una sucursal de una compañia extranjera dedicada al ramo de los seguros, aunque tal denominación no conste en su registro.

Ahora bien, ya hemos señalado más arriba que también existian sociedades en las que los seguros no eran sino una actividad más dentro de sus negocios. Fue el caso, por ejemplo, de "Nau y Palmarini". Registrada en Guipúzcoa en 1892, fue fundada por el propietario afincado en Nueva York Martín Simón Jules Nau y el abogado francés residente en San Sebastián Alphonse Asguasciati Palmarini con un capital de 5.000 ptas. Su objeto era, además de suministrar informes sobre comerciantes de España y Portugal y sobre negociantes extranjeros, la realización de operaciones de banca, cambio o bolsa, ésta última al contado y a plazos, bien por

3. RMG, Lib. 13, Hoja 229.

33 RMG, Lib. 5. Hoja 53. 
medio de la Caisse Mobilière de París, bien por medio de otras personas o casas; la representación en España de sociedades extranjeras, sobre todo de seguros, americanas, inglesas o francesas; o la representación de compañías españolas en las Vascongadas ${ }^{34}$. Como puede observarse, se trata de una sociedad dedicada a todo tipo de actividades comerciales, en el sentido amplio de la palabra, siendo, por consiguiente muy difícil saber qué papel desempeñaban los seguros en el conjunto de las mismas. Otro tanto podemos decir de la compañía "Fausto Echevarría e Hijo", domiciliada en San Sebastián en 1894. Comerciantes de profesión, Fausto y Feliciano Echevarría diversificaron igualmente sus quehaceres comerciales, sin especializarse única y exclusivamente en la contratación de seguros. Asi, esta sociedad se dedicó al comercio de hierros, a la consignación de vapores y a la representación de compañias de seguros y comisiones, manteniendo su actividad hasta 1899, momento en que quedó disuelta al haber fallecido Fausto Echevarria a finales del año anterior ${ }^{35}$. No obstante, su espíritu emprendedor les llevó a participar igualmente en otras empresas de signo bien distinto. Por ejemplo, en 1891 se constituyó en San Sebastián la sociedad anónima "La Prudencia" con el fin de llevar a cabo la explotación de varias minas situadas en las provincias de León y Asturias. En esta compañía, en la que tomaron parte personajes donostiarras tan importantes como el marqués de Rocaverde o Brunet, Feliciano Echevarría figuraba como suplente del consejo de administración y Fausto Echevarría aparecía como accionista con una pequeña participación ${ }^{36}$. Además, Feliciano Echeverría formó parte de la sociedad colectiva «A. Diaz y cía." fundada en la capital donostiarra en 1893 con un capital de 42.337 '95 ptas. y dirigida a la compra-venta de pianos, instrumentos de música y sus accesorios correspondientes. Ese mismo año se constituyó la sociedad anónima "Empresa de La Voz de Guipúzcoa" dedicada a la explotación del periodo republicano del mismo nombre. De las 146 acciones de 250 ptas. cada una Feliciano Echevarría aparecía como suscriptor de siete títulos. Tres años más tarde dicha sociedad se transformó y pasó a denominarse "Sociedad arrendataria de La Voz de Guipúzcoa", consagrada al usufructo del contrato de arrendamiento de La Voz de Guipúzcoa. En este caso, de las 300.000 ptas. de capital social distribuidas en 300 acciones de 1.000 ptas. cada una, la aportación de Feliciano Echevarría ascendió a las 4.000 ptas. También estuvo presente en la primera comisión 
gestora del «Banco Guipuzcoano» en 1899 y en su primer consejo de administración ${ }^{37}$.

Por consiguiente, y según lo expuesto hasta aquí, podemos decir que este subperíodo correspondiente a los años 1886-1900, se caracterizó por la inexistencia de compañias de seguros propiamente donostiarras, aunque el capital local no fue ajeno, ni muchísimo menos, a un negocio que estaba empezando a dar sus primeros pasos. Lo que parece apreciarse, sin embargo, es una clara falta de riesgo, bien recurriendo a la táctica de la representación de otras sociedades con cierta implantación en el campo de los seguros (como los casos de "Pagola y Sagasti", "R. Instander y cía.» e "Hijos de Lapazarán»), bien incorporando este incipiente negocio al conjunto de sus actividades ( Nau y Palmarini» y «Fausto Echevarría e Hijo»). Es preciso señalar que para las profesoras Matilla y Frax, el periodo comprendido entre 1860 y 1899 es de estancamiento, con unas compañias que aparecian y desaparecian, debido, probablemente, a su débil estructura ${ }^{38}$. No obstante, las posibilidades del seguro privado quedaron de manifiesto no sólo por el hecho de que «El Fénix» de París llegara a instalar una sucursal en San Sebastián en una fecha tan temprana como 1887, sin duda por detectar los grandes cambios que la economía y la sociedad guipuzcoanas estaban experimentando en estos momentos gracias al desarrollo de los sectores secundario y terciario ${ }^{39}$, sino también por los pasos más decididos y decisivos dados en este terreno en los primeros años de este siglo 40 .

\section{AFIANZAMIENTO CAPITALISTA Y SEGUROS (1900-1914)}

Efectivamente, entre 1900 y 1914 hemos detectado una serie de cambios que es preciso mencionar ${ }^{41}$. En primer lugar, la novedad más importante fue la aparición de varias sociedades de seguros propiamente donostiarras, en el sentido expresado anteriormente. En concreto, de las seis que se registraron en los catorce primeros años de este siglo, cuatro eran de ese tipo.

\footnotetext{
37 RMG, Lib. 13, Hoja 189: "A. Díaz y cia."; Lib. 12, Hoja 202: "Empresa de La Voz de Guipúzcoa"; Lib. 14, hoja 275: "Sociedad arrendataria de la Voz de Guipuzcoa"; y Lib. 16, Hoja 345: "Banco Guipúzcoano".

Matilla y Frax Rosales (1993).

Véase Garate y Martin Rud (1995).

No debemos olvidar al respecto que en 1908 fue promulgada la Ley General de Seguros, tal y como se ha indicado más arriba.

41 Véase Matilla y Frax Rosales (1993).
} 
La creación en 1901 de la compañía de seguros anónima "El Norte" constituye, sin duda, el dato más importante de todo nuestro período de estudio. Tenía por objeto la realización de seguros marítimos, de incendios, de valores e incluso de vida, accidente laboral y otros. Era, pues, una sociedad que nacía con vocación de dedicarse a todos los aspectos de la actividad aseguradora. Con sus cinco millones de capital social, «El Norte" figuraba a la cabeza de cuantas sociedades se registraron en ese año no sólo en San Sebastián, sino también en Guipúzcoa. Aunque es preciso señalar que de las 2.000 acciones emitidas de 250 pts. cada una, únicamente exigió cubrir ese año el $10 \%$, lo cual equivaldría a 500.000 ptas., disponiéndose que el resto fuera cubierto con posterioridad. Sin embargo, no parece que se cumplieran las expectativas, pues el 1. de agosto de 1911 el capital habia sido reducido a cuatro millones distribuidos en 2.000 acciones de 200 ptas..$^{42}$. La cifra es realmente importante si tenemos en cuenta que ninguna de las cincuenta sociedades registradas en Guipúzcoa en 1911 alcanzaba dicho capital. Aún más, el capital medio de dichas compañías vendría a ser de $613.386,66$ ptas. ${ }^{43}$. El dato puede ser todavía más significativo si tenemos en cuenta que el capital registrado en toda España en 1911 en el ramo de seguros (contra incendios y sobre la vida) alcanzó los 10.223.500 millones de pesetas repartidos en seis sociedades ${ }^{44}$.

«El Norte» fue fundada por algunos de los personajes más destacados del panorama económico de la ciudad de estos años, los socios José L. de Moyúa Leal, propietario, como apoderado de la compañía de seguros de Bilbao "Aurora»; Lucas Garcia Ruiz, propietario, en concepto de director gerente; y dos de los comerciantes más activos de San Sebastián de esta época, Ignacio Mercader Echániz y Ramón Machimbarrena Echave.

Merece la pena, pues, detenernos un momento en los socios de estas compañias, dada su importancia en la economía guipuzcoana de este momento. Así, José Leopoldo de Moyúa fue socio fundador en 1899 y accionista de la "Compañía Electro-química lbérica" que, en contra de los previsto cerró sus puertas en 1901. También formó parte de la "Sociedad Española de envases cilíndricos" de madera (1899-1907), la cual, tras va-

42 RMG, Lib. 17, Hoja 402.

4i Cifra obtenida de dividir 30.669 .333 ptas. desembolsados ese año entre las 50 sociedades registradas (datos extraidos del Anuario de los Registros y del Notariado, año 1911 (1912). Dirección General de los registros y del Notariado, Madrid).

4. Ibidem, pág. 114. 
rios avatares, se vio obligada a disolverse por la pérdida total del capital, dos millones en acciones y 469.000 ptas. en obligaciones ${ }^{45}$. De Lucas Garcia Ruiz, quien fue director del Banco Guipuzcoano a primeros de siglo, sabemos que participó en parte del primer consejo de administración de la sociedad anónima "Fomento de San Sebastián» ${ }^{46}$. Por su parte, Ignacio Mercader ${ }^{47}$, entre 1869 y 1886 , había formado parte con su padre Francisco Luciano de la sociedad «Mercader e Hijos" y, una vez disuelta, había constituido con su hijo Manuel la sociedad colectiva "Mercader e Hijo" en 1886. Ahora bien, paralelamente a sus negocios familiares, Ignacio Mercader figuró igualmente como socio fundador del «Banco Guipuzcoano" en 1899, del cual fue el presidente de la primera comisión gestora y vocal del primer consejo de administración. No hay que olvidar que el «Banco Guipuzcoano» y «El Norte» son dos de las más grandes empresas llevadas a cabo en San Sebastián durante estos años. Finalmente, José Machimbarrena se nos presenta como un representante típico de la burguesía emprendedora donostiarra de la época, ávido de inversiones en los ramos más diversos. Se dedicó a la actividad comercial por medio de diversas compañías ${ }^{48}$; invirtió en sociedades industriales ${ }^{49}$, de espectáculos y fomento ${ }^{50}$; fue socio fundador, vocal de la primera comisión gestora y miembro del primer consejo de administración del Banco Guipuzcoano ${ }^{51}$; fundó su propia sociedad dedicada a la hosteleria ${ }^{52}$ y, como se ha dicho, fue socio constituyente de la aseguradora "El Norte".

Por primera vez nos encontramos con algunos miembros de la élite burguesa donostiarra, como pueden ser Ignacio Mercader y Ramón Machimbarrena, que decidieron invertir en una actividad que, en cierto modo, podemos denominar novedosa, pero con unas perspectivas sumamente interesantes, tal como parece derivarse del gran montante de dinero colocado en esta sociedad.

\footnotetext{
45 RMG, Lib. 15, Hoja 329: "Compañia Electro-química Ibérica", y Lib. 16, Hoja 361 : "Sociedad Española de envases cilindricos".

4f RMG, Lib. 18, hoja 431.

47 RMG, Lib. 3, hoja 25: "MeRCADER e Hijos", Lib. 4, Hoja 26: "MeRCAder e Hijo" y Lib. 16, Hoja 345: "Banco Guipuzcoano".

48 RMG, Lib. 9, Hoja 120: "Blasco y Machimbarena", 1881-1892; y Lib. 10, Hoja 163: "MachimbarRena Hermanos" (1892).

49 RMG, Lib. 11, Hoja 163: "La Maquinista Guipuzcoana", socio de la misma por si y por "MAChIMBARRENA Hermanos" y "OSACAR Hermanos" y accionista; y Lib. 11, Hoja 167: "Compañia de asfaltos Maestu".

so RMG, Lib. 11, Hoja 174: "J. Arana y cia." (1892-1909), donde de un capital de 450.000 ptas. aportó 150.000; Lib. 12, Hoja 195: "ARANA, UNiBASO y cia."; y Lib. 18, Hoja 431: "Fomento de San Sebastián".

5. RMG, Lib. 16, Hoja 345.

52 RMG, Lib. 22, Hoja 717: «R. MachimbarRENA y cia" (1909).
} 
Con todo, “El Norte» no fue la única compañia que rompió con lo que había sido hasta entonces la tónica general del negocio. Es preciso mencionar igualmente a la sociedad "Franco-Inglesa", registrada en San Sebastián en 1904 y que tenía por objeto la contratación de seguros a prima fija de vida, infantiles, retiros, rentas vitalicias, etc. Fue creada por los socios Eusebio Martínes Brasvissiks, ingeniero afincado en Astillero (Santander) y Alfredo Sánchez López, empleado residente en San Sebastián. Contaba con un capital social de un millón de pesetas repartidas en dos mil acciones de 500 ptas., siendo nominativas hasta el $50 \%$ del desembolso, aunque se establecia que posteriormente podrian ser convertidas en acciones al portador. Se estipulaba, además, un desembolso a plazos, con un $40 \%$ hasta el primer trimestre y completándose el resto según las necesidades de la empresa ${ }^{53}$.

Para comprender mejor este tipo de inversiones debemos recurrir al contexto general de la economía guipuzcoana de estos primeros años del siglo $x x$. Tras un primer impulso inversor, comprendido entre los años 1876 y 1898, San Sebastián vivió una etapa de consolidación del capitalismo ${ }^{54}$. Según se observa en el cuadro n. 2 durante la primera década del presente siglo las inversiones crecieron a un ritmo realmente acelerado en el conjunto de la provincia guipuzcoana. Aunque es preciso tener en cuenta la triple distinción que M. Gárate y J. Martín Rudi establecen entre: "a) el número de sociedades creadas, b) las que realmente movilizan recursos - cuando menos guipuzcoanos - y c) las que en definitiva llegan a realizar operaciones" ${ }^{55}$. Las ventajas económicas proporcionadas por el Concierto Económico favorecían la inscripción de empresas que ciertamente no operaban en la provincia ${ }^{56}$. Con todo, éste no parece ser el caso de las compañías aseguradoras de este momento. Más bien cabe pensar que las inversiones en este apartado siguieron el mismo ritmo que el resto de los sectores. A tenor de los trabajos citados y el del profesor Jordi Catalán ${ }^{57}$, se puede constatar que entre 1900 y 1914 no sólo aumentó el volumen de capital invertido en Guipuzcoa, sino que también

${ }_{53}$ Este suele ser uno de los grandes problemas que presenta la documentación obtenida de los registros mercantiles, ya que, al hacer la inscripción de una sociedad, el capital social que figura no siempre se cubre en el momento, sobre todo en las anónimas, siendo muy difícil posteriormente llegar a conocer si se llegó a completar en su totalidad o no, como sucede en el caso de esta compañía.

54 Gámate y Martin Rudi (1995), cap. 5

55 Ibidem, pág. 133. Puede completarse esta visión con la ofrecida por Castells (1987) págs. 38-47 a propósito de los datos extraídos del Registro Mercantil de Guipuzcoa.

56 AlONSO OLEA (1995).

57 CATALAN (1990). 
asistimos a una mayor diversificación de las inversiones. De hecho, la repatriación de capital americano tras la crisis de 1898, las sucesivas renovaciones del Concierto y la Ley de Bases Arancelarias de 1906 contribuyeron decididamente a la expansión económica de la provincia. Por poner un ejemplo próximo, en Vizcaya, y con dimensiones bien distintas, esta diversificación había tenido lugar en la última decada del siglo pasado ${ }^{58}$. Guipúzcoa asistió, pues, durante estos primeros años del presente siglo a un importante proceso no sólo de industrialización, que a la postre marcaria su propia modernización, sino también de terciarización, especialmente su capital. Es precisamente en este marco general de análisis donde debemos situar la nueva fase iniciada por las compañias de seguros a comienzos de este siglo, las cuales empezaron a ser vistas como un negocio más donde invertir, y siempre teniendo en cuenta las paulatinas transformaciones que la sociedad guipuzcoana estaba experimentando en estos momentos.

Cuadro n. 2. Sociedades mercantiles en Guipúzcoa entre 1891 y 1915

\begin{tabular}{lcrrr}
\hline QUINQUENIO & NÚMERO & CAPITAL & N. IN INICE $^{\prime}$ & MEDIA \\
\hline $1891-1895$ & 151 & 28.678 .430 & $100^{\prime} 00$ & $189923^{\prime} 37$ \\
$1896-1900$ & 137 & 26.533 .405 & $92^{\prime} 52$ & $193674^{\prime} 48$ \\
$1901-1905$ & 174 & 67.041 .711 & $233^{\prime} 77$ & $385297^{\prime} 18$ \\
$1906-1910$ & 247 & 135.069 .609 & $470^{\prime} 97$ & $546840^{\prime} 52$ \\
$1911-1915$ & 283 & 122.797 .753 & $428^{\prime} 18$ & $433914 ' 32$ \\
\hline
\end{tabular}

FUENTE: Castells (1987), pág. 45 y Luengo (1988), pág. 102, a partir de las estadisticas elaboradas por la Dirección General de los Registros y del Notariado.

Pero además de "El Norte» y la «Franco-Inglesa", hubo otras dos compañias propiamente donostiarras dedicadas al seguro privado durante estos años, aunque de dimensiones mucho más modestas. Nos estamos refiriendo a "La Previsión Popular" y a "Sagasti e Hijo». Así, entre 1910 y 1914 funcionó en la capital donostiarra "La Previsión Popular», una sociedad anónima dedicada al seguro de enfermedad, invalidez y muerte ${ }^{59}$. Estuvo constituida por el jubilado Manuel Ezquerra, por el militar Joaquín 
Usunáriz y por los comerciantes Antolín Alvaro Palencia y Nicolás Usabiaga, todos ellos domiciliados en San Sebastián. Su capital social fue de 40.000 ptas. dividido en 400 acciones de cien pesetas cada una, de las cuales Ezquerra llegó a suscribir cincuenta y los demás diez cada uno. Con todo, en 1912 se llevó a cabo una reducción de capital, concretamente de siete mil pesetas que representaban las acciones de Dolores de Mocoroa y Ramón Bernaondo, que sumaban sesenta y diez títulos respectivamente. Por su parte, la segunda de la compañías mencionadas, "Sagasti e Hijo", fue constituida por José Antonio Sagasti, quien, como ya se ha dicho más arriba, formó parte de «Pagola y Sagasti», y su hijo, el abogado Juan Antonio Sagasti ${ }^{60}$. Debemos resaltar el hecho de que por primera vez un socio de una sociedad de este tipo se autodenomina "asegurador", siendo lo común hasta entonces figurar con la profesión de "comerciante". Otro dato curioso es que se creó sin capital alguno, confiando posiblemente en la actividad que José Antonio Sagasti llevaba realizando en esta plaza desde mediados de los ochenta del siglo pasado. En este sentido retomamos la idea mencionada anteriormente de la creación de una especie de tradición familiar en algunos casos, como es éste que ahora nos ocupa.

Junto a estas compañías, hemos de señalar igualmente la fundación en 1914 de "A. Vildósola y cia.", dedicada a las operaciones de seguros para la compañia "L'Abeille» u "otra análoga» ${ }^{61}$. Por lo tanto, se creaba como representación de otra sociedad. Podemos decir que ésta fue la única de estas características de este momento. Esta puede ser, sin duda, otra de las diferencias importantes respecto del subperiodo anterior, donde fueron varias las compañías que actuaron en representación de otras. Con un capital de 1.500 ptas., sus socios fundadores fueron el industrial de Hernani Antonio Vildósola y el comisionista de San Sebastián Lorenzo Pérez Peña. No fue éste, ni mucho menos, el único negocio al que se dedicaron ambos inversores. Ese mismo año de 1914 Antonio Vildósola fundó junto con Florentino Azqueta la sociedad colectiva "Vildósola-Azqueta" dedicada a la explotación de un negocio de motocicletas, reparación de las mismas, venta de gasolina y accesorios y todo lo relacionado con esta actividad y con un capital social de 20.000 ptas. ${ }^{62}$. Por su parte, Lorenzo Pérez figura en varias iniciativas empresariales de estos años. Aparecía como socio en la disolución en 1900 de "Henri Garnier y cía.", dedicada a la elaboración

RMG, Lib. 24, Hoja 865.

RMG, Lib. 25. Hoja 1.022 .

RMG, Lib. 25, Hoja 1.018. 
de licores. En la nueva «H. Garnier y cía., sociedad en comandita", Pérez Peña desempeñó el cargo de gerente junto a Garnier hasta su desaparición en 1903, año en que se creó la sociedad anónima «Destilerías H. Garnier», donde también figuraba como accionista ${ }^{63}$.

Por último, y como sucediera en el primer subperíodo, también en estos años nos encontramos con una sociedad para la cual los seguros no eran sino una actividad más dentro de sus operaciones. Fundada en 1912, nos referimos al "Banco Español del Río de la Plata", de cuyos datos conocidos no podemos derivar si realmente tuvo alguna participación en este ramo ${ }^{64}$. Este banco, radicado en Buenos Aires, existía desde el Decreto del Gobierno Nacional del 1. de septiembre de 1886, momento en que fueron aprobados sus estatutos. Su objeto, además de la realización por cuenta propia o por terceros de toda clase de operaciones bancarias, era constituir o formar otras sociedades de crédito, de seguros, hipotecarias, inmobiliarias, dedicadas a la construcción o a la realización de obras públicas. Mientras el Banco se constituyó con un capital de cien millones de pesos divididos en un millón de acciones de cien pesos cada una, la sucursal de San Sebastián se creó con un capital de un millón de pesetas y se estableció que se rigiera por los mismos estatutos que el Banco Español y que sus operaciones se adecuasen a la legislación española.

Por consiguiente, podemos decir que fue en estos primeros años del presente siglo cuando las compañías aseguradoras dieron sus primeros pasos firmes hacia la consolidación de un subsector cada vez más rentable, tal como habria de verse sólo unos años más tarde ${ }^{65}$. Indudablemente, el hecho de que surgieran sociedades dedicadas únicamente a esta actividad no hace sino avalar esta afirmación. De esta forma, pues, quedaban fijadas de manera sólida las bases que habrían de cimentar un negocio cada vez más próspero y floreciente.

\section{CONCLUSIONES}

Todavía vivos los postulados de los revolucionarios franceses y las disposiciones de las Cortes de Cádiz, el Liberalismo fue asentándose en

3 RMG, Lib. 16, Hoja 365 y 366 y Lib. 18, Hoja 466.

64 RMG, Lib. 24, Hoja 864; Album gráfico descriptivo del pais Vascongado. Años 1914-15. San Sebastián, 1915; Garate (1994), págs. 129-130 y Gárate y Martin Rudi (1995), págs. 220 221.

65 Para España en general, Matilla y Frax Rosales (1993). 
España a lo largo de la primera mitad del siglo xIx, desmoronándose poco a poco las instituciones, estructuras, características y ligazones propias del Antiguo Régimen. Como parte constitutiva del mismo, el concepto de propiedad adquirió entonces unas dimensiones hasta entonces inéditas, de suerte que no resulta extraño que las primeras iniciativas en este terreno se centrasen en el seguro de propiedades, lo cual, y pese a constituirse en forma de mutuas, resultaba un verdadero signo de modernidad. Porque frente a la mutua de carácter gremial, y concebida como una especie de beneficiencia solidaria y grupal, nos hallamos con unas mutuas de carácter totalmente distinto, tal como se ha podido comprobar. Ahora bien, y pese a ser los antecedentes más directos de las compañías privadas de seguros, es preciso decir que existían sensibles diferencias entre ambos tipos de sociedades. Así, mientras aquellas "asociaciones" aseguraban sólo propiedades, tanto urbanas como rurales, las compañias trabajaban prácticamente con inmuebles urbanos, a la vez que fueron incorporando poco a poco seguros de vida, accidente, invalidez, etc. Posiblemente la élite que en estos momentos empezó a hacer seguros personales jamás pensó en una mutua para llevarlos a cabo. No debemos olvidar que el porcentaje de población capaz de acceder a ellos disfrutaba de un nivel económico alto.

Fue, pues, a partir de los años ochenta del siglo XIX cuando se crearon estas primeras compañías de seguro privado en Guipúzcoa. Sin un marco legislativo propio y con las características precauciones de una actividad incipiente, fue preciso esperar a comienzos de este siglo para que los seguros empezaran a concebirse como un negocio más. Con un capitalismo cada vez más asentado y en plena expansión económica, se detecta una diversificación de las invesiones que no sólo afectó a nuevos ramos industriales, sino también al sector terciario, como éste que nos ocupa. A partir de 1900, pues, y al igual que sucediera en toda España, nos encontramos con los primeros pasos sólidos y firmes de un subsector llamado a un próspero futuro en la segunda mitad de los años diez y siguientes. Indudablemente, no podemos entender el desarrollo de esta ocupación sin contextualizarlo en el marco general de las transformaciones que la sociedad guipuzcoana experimentó durante estas décadas. En una sociedad totalmente capitalista, la previsión personal fue ganando cada vez mayor fuerza frente a una beneficiencia que, en semejante contexto, estaba llamada a desaparecer. No es sorprendente, pues, que las compañias privadas empezaran a prestar este tipo de servicios, si bien no todas las clases sociales se pudieron costear su propio seguro. De ahí que el Instituto Nacional de Previsión paliara, en buena medida, esta deficiencia. 


\section{BIBLIOGRAFIA}

Album gráfico descriptivo del pais Vascongado. Años 1914-15 (1915). San Sebastián

Alonso OLEA, Eduardo (1995): El Concierto Económico (1878-1937). Origenes y formación de un Derecho histórico, IVAP, Oñate.

Anuario de los Registros y del Notariado, año 1911 (1912), Dirección General de los Registros y del Notariado, Madrid.

ApARICIO, Celia (1991): Poder municipal, economia y sociedad en la ciudad de San Sebastián (1813-1855), Instituto Dr. Camino de Historia donostiarra, San Sebastián.

BARCENILla, Miguel Angel (1992): La primera industrialización de la comarca de Renteria, 18451905. Tesis doctoral inédita, UPV.

CASTELLS, Luis (1987): Modernización y dinámica politica en la sociedad guipuzcoana de la Restauración, 1876-1915. Siglo xxI y UPV, Madrid.

CASTILLO, Santiago (1996) (ed.): Solidaridad desde abajo. Trabajadores y Socorros Mutwos en la España Contemporánea, UGT. Madrid.

CATALAN. Jordi (1990): "Capitales modestos y dinamismo industrial: origenes del sistema de fábrica en los valles guipuzcoanos, 1841-1918", en NADAL, J. y CARRERAS, A. (eds.): Pautas regionales de la industrialización española (siglos $x i x$ y $x x$ ). Ariel, Barcelona.

Cipolla, Carlos M. (2 ${ }^{-}$ed. 1992): Historia económica de la Europa preindustrial, Alianza, Madrid.

Cruz Mundet, José Ramón (1991): Renteria en la crisis del Antiguo Régimen (1750-1845). Familia, caserio y sociedad rural, Ayto. de Rentería, Rentería.

GARATE, Montserrat (1976): El proceso de desarrollo económico en Guipúzcoa, Cámara de Comercio, Industria y Navegación de Guipúzcoa, San Sebastián.

(1994): “Instituciones financieras en el Pais Vasco. Articulación y crecimiento del sistema bancario en Gipuzkoa (1899-1930)", en MARTin ACEÑA, P. e Idem: EConomia y empresa en el Norte de España (Una aproximación histórica), Diputación Foral de Guipúzcoa y UPV, San Sebastián.

Garate, M. y Martín Rud, Javier (1995): Cien años de la vida económica de San Sebastián (1887-1987), Instituto Dr. Camino de Historia donostiarra, San Sebastián.

GARRIDO y COMAS, J.J. (1994): El mutualismo privado en la Europa del siglo xx. Mapfre, Madrid.

González Portilla, Manuel (1981): La formación de la sociedad capitalista en el Pais Vasco. Haranburu, San Sebastián.

LUENGO, Félix (1988): "El proceso de modernización económica de Guipúzcoa a través de la formación de sociedades mercantiles (1886-1923)", en /1" Congreso Mundial Vasco, t. V. Gobierno Vasco, Vitoria.

MARTINEZ QuINTERO, M.E. (1990): “La fundación del INP. Las primeras experiencias de previsión social", en MONTERO GARCia, F.: Origenes y antecedentes de la previsión social. Los Seguros Sociales en la España del sigio xx. Instituto Nacional de Previsión, Madrid.

MATILLA, M. J Jesús (1986): "La regulación del sistema capitalista en España (1829-1923): la constitución de las sociedades por acciones", Estudios de Historia Social, n. " 38-39.

Matilla, M. J. y Frax Rosales, Esperanza (1993): "Los seguros en España, 1830-1934", V Congreso de la Asociación de Historia Económica, San Sebastián.

Montero, Manuel (1990): Mineros, banqueros y navieros, UPV, Lejona (Vizcaya).

(1994): La burguesia impaciente. Especulaciones e inversiones en el desarrollo empresarial de Vizcaya, Beitia, Bilbao.

PESET, J.L. (1978): "Capitalismo y medicina: ensayo sobre el nacimiento de la Seguridad Social", Estudios de Historia Social, n. 98.

Reglamento para la Sociedad de Seguros Mutuos de Casas y Caserios de Guipúzcoa (1848), Imprenta de A. Gorosábel. Tolosa. 